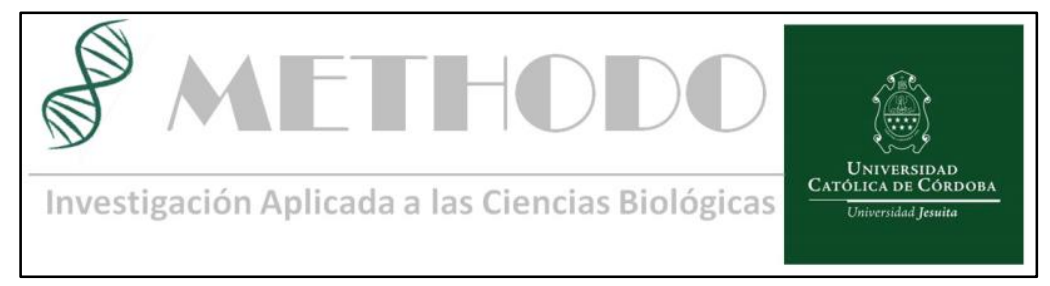

ARTICULO de REVISION Methodo 2017 Nov;2(1):03-15 DOI: $10.22529 / \mathrm{me} .2017 .2(1) 02$

Recibido 23 Feb 2017 | Aceptado 10 Abr 2017| Publicado 30 Abr 2017

\title{
Mecanismos de la conexión nariz-pulmón
}

\section{Mechanisms of nose-lung connection}

\author{
Saranz $\mathrm{RJ}^{1}$, Lozano $\mathrm{A}^{1}$, Lozano $\mathrm{NA}^{1}$, Sosa Aguirre $\mathrm{AG}^{1}$, Alegre $\mathrm{G}^{1}$.
}

\section{Resumen}

La vía respiratoria, de la nariz al pulmón, actúa como un sincicio anátomo -funcional con su correlato fisiopatológico. Desde tiempos remotos los autores estudiaron los mecanismos de conexión entre la vía respiratoria superior e inferior que pudiera explicar la elevada coincidencia en la prevalencia de sus respectivas patologías.

Algunos de esos mecanismos no se han podido demostrar fehacientemente y las investigaciones muestran resultados contradictorios. Otros responden a determinados fenotipos como el de la relación entre rinitis y asma alérgicas, el modelo más estudiado, en el que un fenómeno inflamatorio mediado a través del torrente circulatorio es el más aceptado. No obstante los fenotipos de enfermedad respiratoria no alérgica y el de la patología mediada por lgE local en nariz y bronquios, no tienen una conexión absolutamente resuelta por las investigaciones.

En esta revisión discutiremos los distintos mecanismos de interacción nariz pulmón que por sus características anatómicas, fisiológicas y fisiopatológicas avalan la hipótesis de "una vía aérea única, una única enfermedad" aceptada en la actualidad por la mayoría de los autores.

Palabras claves: Rinitis, asma, vía aérea superior, vía aérea inferior, interacción nasobronquial

\footnotetext{
Abstract

The airway, from the nose to the lung, may be accepted as an anatomo-functional unit with its physiopathological correlate. From a long time ago the authors studied the mechanisms of connection between the upper and lower airway that could explain the high coincidence in the prevalence of their respective pathologies.

Some of these mechanisms have not been proven conclusively and the research shows contradictory results. Others respond to certain phenotypes such as the relationship between rhinitis and allergic asthma, the most studied model, in which an inflammatory phenomenon mediated through the bloodstream is the most accepted. However the knowledge of phenotypes of non-allergic respiratory disease and the pathology mediated by local $\lg E$ in the nose and bronchi, they do not have a connection mechanisms completely determined by the investigations.

In this review we will discuss the different mechanisms of nasal lung interaction that support its anatomical, physiological and pathophysiological characteristics responsible for "one airway, one disease" currently accepted by most authors.

Keywords: Rhinitis, asthma, upper airway, lower airway, nasobronchial interaction

1.Universidad Católica de Córdoba, Argentina, Facultad de Medicina, Cátedra de Inmunología. Clínica Universitaria Reina Fabiola, Servicio de Alergia e Inmunología Correspondencia: Ricardo J. Saranz. Servicio de Alergia e Inmunología- Clínica Universitaria Reina Fabiola. Oncativo 1248 -X5004FHP- Córdoba, Argentina. e-mail: rsaranz@arnet.com.ar
}

Revista Methodo: Investigación Aplicada a las Ciencias Biológicas. Facultad de Medicina. Universidad Católica de Córdoba. Jacinto Ríos 571 Bo Gral. Paz. X5004FXS. Córdoba. Argentina. Tel.: (54) 351 4517299 / Correo: methodo@ucc.edu.ar / Web: methodo.ucc.edu.ar | ARTICULO de REVISION Methodo 2017 Nov;2(1):03-15 


\section{Introducción}

La relación entre la vía aérea superior e inferior ha sido observada desde tiempos remotos, pero el concepto de una "vía aérea única" ha sido ampliamente investigado y difundido en las últimas dos décadas a través de guías clínicas específicas $(1,2)$.

Esto implica que nariz, oído medio, senos paranasales y laringe se relacionan con la vía aérea intratorácica como una unidad anátomo-funcional que tiene directas implicancias fisiológicas, fisiopatológicas, clínicas y terapéuticas (3).

Ese modelo ha sido mejor estudiado con la relación existente entre rinitis y asma (3-8). La exacerbación de la enfermedad en un área como la nariz, tiene impacto sobre la afección pulmonar y viceversa, lo que incrementa los costos sanitarios para su control $(1,2,9)$.

El nexo anátomo-funcional entre las vías aéreas superior e inferior también es sustentado por la evidencia de que pacientes con rinitis y sinusitis, sin asma, manifiestan alteraciones subclínicas en su vía aérea inferior expresadas como hiperreactividad bronquial , función pulmonar anormal, e inflamación endobronquial que no tienen correlación sintomática y que potencialmente pueden contribuir a aumentar la morbilidad respiratoria (1015).

La rinitis, por su naturaleza crónica está característicamente asociada con otras entidades mórbidas que incluyen conjuntivitis alérgica, sinusitis infecciosa, poliposis nasal, otitis media con efusión y asma (16). La mayoría de estas enfermedades asociadas pueden justificarse por la vecindad anatómica entre las cavidades nasales y los órganos afectados. Sin embargo, su relación con el asma, ampliamente considerada por los autores (3-5), pudiera explicarse por otros mecanismos puesto que la relación anatómica es más distante. Esos mecanismos han sido ampliamente estudiados pero para muchos de ellos los resultados obtenidos por los autores han sido contradictorios (17-20). El objetivo de esta revisión es analizar y describir los posibles mecanismos de interacción nariz-pulmón que sostienen la hipótesis de la "vía aérea unida".

\section{Mecanismos de interacción entre las vías aéreas superior e inferior}

Numerosos mecanismos locales se han considerado para explicar la interacción entre la nariz y el pulmón, pero algunos no están definitivamente aclarados $(3,7,17,19)$. Las teorías sugeridas son la pérdida de la función protectora por la obstrucción nasal, la estimulación de un reflejo naso-sinusobronquial y la propagación del 
contenido inflamatorio desde la nariz

por aspiración al tracto bronquial

(descarga post nasal). Menos

relevantes son la disminución de la respuesta beta adrenérgica bronquial, la alteración del patrón respiratorio y la alteración de la producción de óxido nítrico nasal (3,7,17-19) Si bien estos mecanismos podrían ser comunes para rinitis alérgica y no alérgica, los estudios no aportan la evidencia concluyente para que ellos, por si solos, puedan explicar la conexión nariz-pulmón (7).

El mecanismo inflamatorio sistémico eosinofílico es el más aceptado. El modelo sistémico de enfermedad alérgica, ha establecido el concepto patogénico de diseminación hemática del fenómeno inflamatorio común con manifestaciones clínicas

predominantes y variables según el órgano blanco principalmente afectado $(21,22)$. Este mecanismo indica que el sitio "blanco" final de la reacción alérgica es la vía aérea en toda su extensión, desde la nariz al bronquio y no sólo el área de la misma donde se produce la reacción.

El fenómeno inflamatorio local mediado por inmunoglobulina $E$ ( $\lg E)$ común a nariz y bronquios está adquiriendo mayor relevancia fisiopatogénica $(23,24)$. No obstante, por las evidencias actuales, el mecanismo inflamatorio diseminado por vía hemática no explica el fenómeno de sensibilización mediado por lgE órgano local recientemente descripto para formas de rinitis y asma por lo que el nexo de su interrelación en estas situaciones no está aún aclarado.

\section{Función nasal deficiente y pérdida del efecto protector de la nariz}

La nariz desempeña un rol protector de la vía aérea intratorácica, a través de la capacidad de purificar, humidificar y acondicionar el aire inspirado para que llegue a una temperatura de $37^{\circ} \mathrm{C}$ a los pulmones (25). Además, la mucosa nasal con sus glándulas submucosas forma parte del sistema inmune innato y adaptativo puesto que produce lisozima, lactoferrina, antioxidantes, defensas químicas e $\lg \mathrm{A}$ secretoria, citoquinas y quimioquinas derivadas del epitelio que permiten proteger la vía aérea inferior de virus y bacterias patógenas, irritantes y alergenos. $(3,26)$. En adición, el óxido nítrico, principalmente producido por la mucosa de los senos paranasales, podría tener un efecto protector sobre la vía aérea inferior del humano por su capacidad bacteriostática, antiviral y mejora la oxigenación con capacidad broncodilatadora adicional $(17,25)$. La congestión nasal propia de la rinitis puede afectar total o parcialmente la función nasal. La obstrucción nasal existente obligaría al paciente a una respiración bucal compensadora, 
perdiéndose funciones protectoras naturales. Sería racional considerar que el enfriamiento de la vía aérea inferior puede ser condicionante de una reducción en la función pulmonar en individuos predispuestos con hiperreactividad bronquial o asma (ej.: asma por ejercicio). No obstante, el clípeo nasal en pacientes con rinitis alérgica, no condujo a un aumento de la respuesta asmática ni se observó relación entre el grado de bloqueo nasal inducido por el desafío nasal con alérgenos y cambios en la reactividad bronquial $(22,27)$. Más aún, un reciente estudio considera que la respiración bucal habitual en niños con rinitis alérgica persistente y asma puede reducir el grado de asma inducida por ejercicio (28). Pareciera entonces que el impacto de la respiración bucal sobre la vía aérea inferior no resulta relevante.

\section{Estimulación de un reflejo naso- sinu-bronquial}

Desde principios del siglo XX, se postuló que en la fisopatogenia del asma intervendría un reflejo neural originado en la nariz $(29,30)$. Así se consideró que la estimulación mecánica o química de receptores en nariz, laringe y tráquea puede inducir estornudos, tos y broncoconstricción para prevenir la penetración de alérgenos e irritantes en pulmón (31).
Sluder en 1919 (29) describió la entidad de un reflejo sensorial que discurre por vía aferente trigeminal y eferente vagal y que produce contracción del músculo liso bronquial que ha estado en debate por varios años. En el modelo animal prácticamente no existen dudas de su existencia, pero en el ser humano los datos son controvertidos dado que posteriores estudios aportaron evidencias a favor y en contra de su existencia $(32,33)$.

Con la aplicación de un irritante o un alérgeno en la nariz de individuos noatópicos y atópicos respectivamente, algunos autores demostraron $(34,35)$ y otros no $(36,37)$ un efecto de aumento de la resistencia de la vía aérea inferior. Sin embargo, existió algunas diferencias entre los ensayos: los pacientes incluidos con resultados negativos fueron evaluados en un estado asintomático, mientras que los que arrojaron resultados positivos manifestaban enfermedad nasal activa al momento de ser evaluados. Se ignora el significado fisiopatológico de estas diferencias pero un umbral crítico de síntomas nasales puede ser requerido para que la estimulación nasal provoque cambios en la vía aérea intratorácica (38). En 1969 Kaufman y cols. (34), aplicando partículas de sílice en la mucosa nasal de sujetos sin asma 
encontró un significativo e inmediato incremento en la resistencia de las vías aéreas, que fue bloqueado por la administración sistémica de atropina y la resección trigeminal, sugiriendo un efecto mediado por un reflejo colinérgico.

La exposición nasal al aire frío puede causar broncoconstricción en sujetos con asma (39). No obstante, la aplicación endonasal de histamina en rinitis alérgica perenne arrojó resultados disímiles para la generación de una respuesta inmediata broncoconstrictora (40). Una evidencia (41), aportaría al soporte a la evidencia experimental de que un reflejo nasobronquial es viable en humanos. Usando capsaicina tópica en la nariz, un potente estimulante neural específico, observó que en el 30\% de los sujetos con rinitis y asma ocurrió una rápida reducción en FEV1 y FVC dentro de los 30 minutos de consumado el desafío.

La mayoría de los autores consideran que la aceptación de un reflejo nasobronquial podría ser una teoría simplista, puesto que la estimulación sería episódica y su recurrencia podría inducir taquifilaxia en la respuesta de la vía aérea intratorácica $(8,17,42)$. El fenómeno broncoconstrictor y de hiperreactividad bronquial frecuentemente observado en sujetos con rinitis podría ser potenciado por este reflejo, pero se requieren más estudios que lo confirmen (43).

\section{Descarga de secreciones nasales hacia el pulmón}

Hace ya más de 2000 años Claudius Galenus estudió la vía respiratoria como una unidad "integrada" asumiendo que rinitis y asma eran causadas por la "descarga" de secreciones desde el cerebro hacia nariz y pulmón. Ese concepto inicial fue seguido por la hipótesis de que productos proinflamatorios de las secreciones nasosinusales de pacientes con rinosinusitis son aspirados hacia las vías aéreas inferiores con consecuencias adversas para su función como explicación de la relación nasobronquial (30).

La evidencias actuales sostienes que, si bien es posible que la descarga post nasal de secreciones del tracto respiratorio superior puede explicar la tos de individuos con síntomas rinosinusales agudos o crónicos estimulando receptores irritantes en faringe y laringe, es más cuestionable la teoría de que estas secreciones pueden alcanzar el tracto respiratorio inferior en cantidades tales que generen exacerbaciones o un empeoramiento crónico de la función pulmonar de pacientes con asma (8).

Bardín y cols. (44) fue incapaz de documentar el aspirado de secreciones radiomarcadas desde 
senos paranasales hacia los campos

pulmonares en 13 pacientes con

rinosinusitis y asma dentro de las 24

horas. La aplicación nasal de alérgenos

radiomarcados produjo modificaciones

en la reactividad bronquial con depósito

intestinal del radiomarcado pero sin

presencia demostrable en mucosa

endobronquial (27). Bajo un modelo

experimental en conejos, Brugman (45)

pudo demostrar la importancia de este

mecanismo en el desencadenamiento

de hiperreactividad bronquial, pero este

patrón es difícil de reproducir en

humanos. Por lo tanto la mayoría de los

autores considera improbable que este

mecanismo tenga un rol en la

interacción rino-sinuso-pulmonar.

\section{El fenómeno inflamatorio común con} propagación hematógena sistémica

La rinitis alérgica es una enfermedad inflamatoria consecuencia de la unión alergeno-IgE específica en las cavidades nasales y en la que una amplia gama de células y mediadores proinflamatorios contribuyen a su expresión clínica (46).

Un soporte científico adicional para la relación entre el tracto respiratorios superior e inferior se ha observado en investigaciones que demuestran una conexión inflamatoria entre nariz y pulmón $(21,22)$. Ambos compartimentos están conectados inmuno- patológicamente a través del torrente circulatorio. Muchos pacientes con rinitis alérgica no manifiestan

síntomas de asma. No obstante la vía aérea inferior presenta signos anatomo patológicos de inflamación. El aparato respiratorio se encuentra afectado en su totalidad pero solo existen manifestaciones clínicas a nivel nasal $(3,8,47)$.

Una reacción alérgica producida en la nariz puede inducir infiltración eosinofílica en el endobronquio, con o sin broncocostricción (21). La existencia de células inflamatorias, en especial eosinófilos, fue claramente establecida tanto en pacientes con asma como en sujetos con rinitis polínica (sin asma), aún fuera de la estación, hallazgo más acentuado cuando existió hiperreactividad bronquial (48).

Bajo el modelo de relación entre rinitis y asma alérgicas el nexo fisiopatológico ha sido demostrado por prolijos estudios de Braunstahl $(49,50)$. La provocación con alérgenos en la nariz desencadena una comunicación específica entre la vía aérea y la médula ósea, a través de la señalización de citocinas hematopoyéticas, estimulando la producción de progenitores del linaje eosinófilo-basófilo en la médula ósea. La migración de esas células hacia bronquios, con expresión de moléculas de adhesión celulares como ICAM-1, VCAM-1 y E-selectina podría explicar 
por qué los pacientes con rinitis

alérgica padecen asma o al menos,

inflamación endobronquial e

hiperreactividad bronquial

subclínica (49).

Este proceso puede ser inverso desde bronquio a fosas nasales. El mismo autor bajo un diseño similar (50), demostró que la broncoprovocación segmentaria con polen de gramíneas en sujetos con rinitis alérgica estacional produjo aumento en la degranulación nasal de mastocitos, un incremento en el influjo de basófilos, y simultánea elevación de niveles séricos de IL-5, eotaxin, and eosinófilos en el resto de la mucosa bronquial (no desafiada con el alergenos) y en la mucosa nasal, e impacto en la función nasal y bronquial, efecto no observado en controles sanos.

Estos hallazgos demuestran que el sitio "blanco" final de la reacción alérgica es la vía aérea en su conjunto y no sólo el área de la misma donde se desencadena la reacción, como consecuencia de un mecanismo sistémico inmunológico-inflamatorio, con impacto en médula ósea que podría ser responsable del desarrollo de un fenómeno más general que incluye la vía respiratoria como unidad desde la nariz al pulmón $(21,22)$.

Esto ha sido reproducido con resultados parcialmente contrapuestos en un modelo murino. La inhalación de un alérgeno a nivel nasal en ratones con rinitis alérgica desencadena una comunicación específica entre la vía aérea y la médula ósea que lleva una mayor producción de IL5 y Factor Estimulante de Colonias GranulocitoMacrófagos (GM-CSF, crítico para que se estimule la producción de progenitores del linaje eosinófilobasófilo (51). Por otro lado, en un modelo murino de inflamación alérgica respiratoria inducida por ovoalbúmina, el desafío endotraqueal en ratones, después de la sensibilización intraperitoneal, produjo inflamación e hiperreactividad bronquial pero sin signos de inflamación en la nariz (52). Aun así, el hallazgo de una relación directa entre el grado de eosinofilia periférica con los puntajes clínicos y de tomografía computada sinusal permite especular que rinosinusitis y asma pueden ser expresión de un proceso inflamatorio común en humanos, mediado por vía sistémica, en el que el eosinófilo jugaría un rol central (53). Estas interesantes observaciones, no obstante, no permiten explicar la interacción entre la rinitis y asma no alérgicas. Probablemente se correspondan con endotipos y fenotipos distintos y la patogenia de su interrelación aún no está definitivamente clarificada. Por lo tanto, resta establecer con mayor precisión los mecanismos de conexión entre las 
vías respiratorias superior e inferior en ausencia de alergia y, en particular, si existe algún mecanismo inflamatorio sistémico responsable. detectan lgE "in situ" en mucosa nasosinusal y bronquial en individuos en quienes no se puede demostrar $\lg \mathrm{E}$ alérgeno-específica en piel o suero,

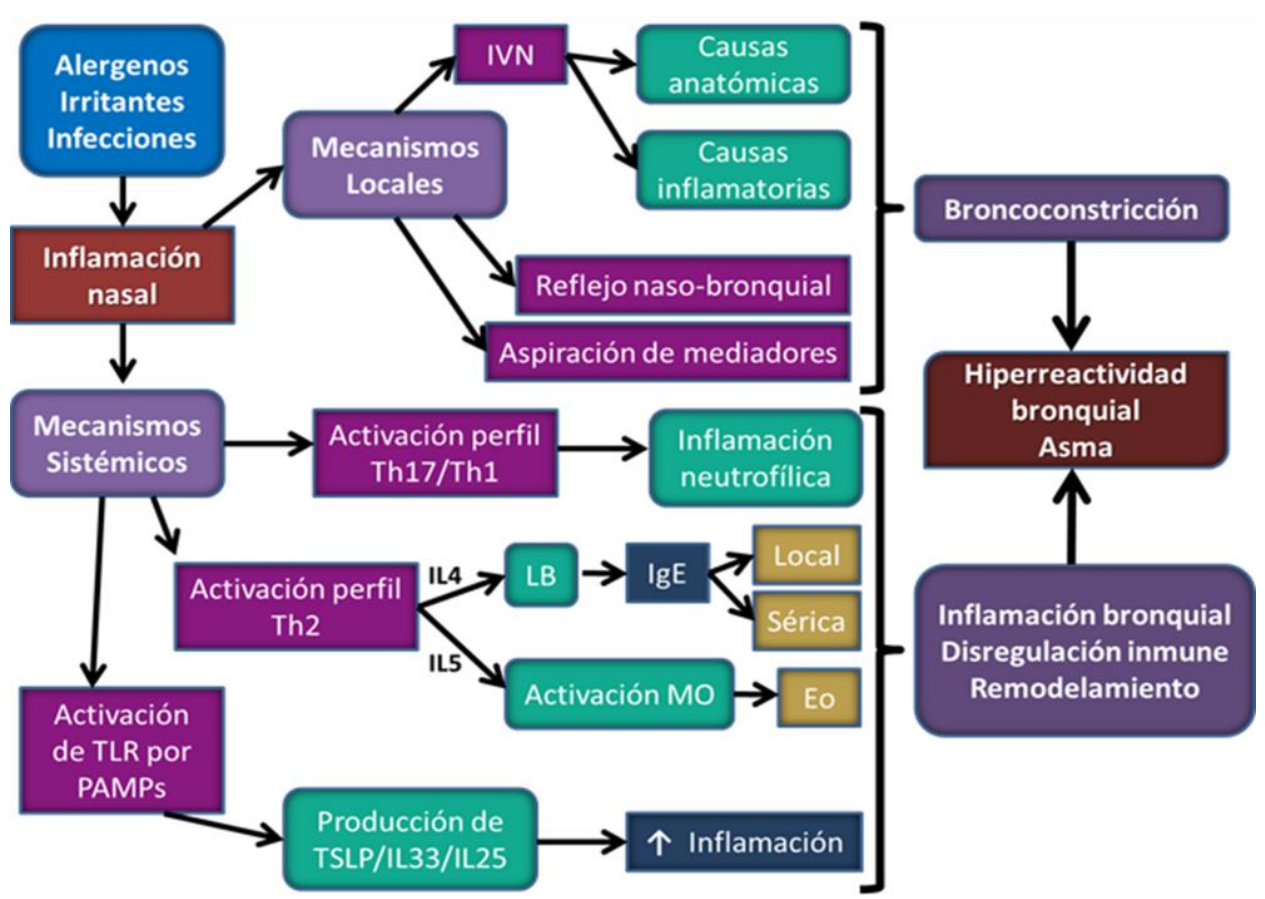

IVN: Insuficiencia ventilatoria nasal; Th: T helper; TLR: toll-like receptor; PAMPs: pathogen-associated molecular patterns; IL: Interleucina; TSLP: thymic stromal lymphopoietin; LB: Linfocito B; IgE: Inmunoglobulina E; MO: Médula ósea; Eo: Eosinófilo.

Figura 1: Potenciales mecanismos de interacción entre vías aéreas superior e inferior. (Tomado de Agresta MF, Saranz RJ, Lozano A, Lozano NA. Relación entre rinitis y asma: ¿Está todo dicho?. Revista de la Facultad de Ciencias Médicas 2014; 71:111-121)

\section{Inflamación local mediada por IgE: cuál es su rol?}

Recientes estudios indican la existencia de rinitis alérgica y asma sin evidencias de sensibilización sistémica demostrable por pruebas cutáneas o métodos "in vitro", fenómeno denominado "entopía" $(23,24,54)$. Así la inflamación local mediada por lgE común a nariz y bronquios, también puede tener un rol patogénico, alejado de la hipótesis de inflamación mediada sistémicamente a través del torrente circulatorio. Nuevos hallazgos aunque su especificidad antigénica y funcionalidad (principalmente en asma) no está definitivamente determinada. Del mismo modo, superantígenos bacterianos, especialmente provenientes de Staphylococcus aureus, jugarían un rol para inducir el cambio de clase de inmunoglobulina con producción de lgE específica en las células $B$ de la mucosa (55). En rinitis, rinosinusitis crónica y en distintos fenotipos de asma aparentemente "no alérgicos" por la imposibilidad de detección de 
sensibilización asociada por los

métodos clásicos, la existencia IgE

local antígeno específica en mucosas

está adquiriendo mayor relevancia

fisiopatogénica y clínica (20). En tal

caso la provocación nasal o bronquial

con alérgenos adquiere significado

diagnóstico y es un verdadero desafío

médico para diferenciar formas no-

alérgicas de rinitis y asma del fenotipo

de "alergia local" $(23,24)$.

En la actualidad no disponemos

estudios de cómo impacta la patología

de vía aérea superior sobre la vía

aérea inferior para el caso de

sensibilización "local". Pareciera que

son fenómenos confinados al órgano

afectado por los que los mecanismos

de interrelación, en las circunstancias

actuales, son difíciles de

determinar (56).

La figura 1 resume algunos de los aspectos patogénicos de la relación entre vías aéreas superior e inferior.

Conclusiones y necesidades por conocer

\section{Bibliografía}

1. Bousquet J, Van Cauwenberge $P$, Khaltaev N, et. al. Allergic rhinitis and its impact on asthma. ARIA workshop report. J Allergy Clin Immunol 2001; 108(5): S147-S334.

2. Bousquet J, Khaltaev N, Cruz AA, et al. Allergic Rhinitis and its Impact on Asthma (ARIA) 2008 Update (in
Las vías aéreas superior e inferior constituyen un único sistema con similitudes en términos de histología, fisiología y en su correlación patológica. Tradicionalmente la relación entre ambas compartimentos anatómicos se estudió bajo el soporte de la enfermedad alérgica respiratoria en que la inflamación sistémicamente mediada es el mecanismo más aceptado. No obstante la existencia de fenotipos no alérgicos y el recientemente descripto "fenómeno alérgico local" nasal y bronquial requiere nuevos estudios para establecer con mayor precisión los mecanismos de interrelación entre las vías respiratorias superior e inferior en ausencia de alergia mediada por vía sistémica. Esto contribuirá a que las expresiones características de rinitis y asma sean abordadas de manera integrada considerando que es probable que diferentes endo-fenotipos de asma se relacionen con las formas de rinitis alérgica y no alérgica. collaboration with the World Health Organization, GA2LEN and AllerGen). Allergy 2008; 63: S8S160.

3. Fasano MB. Combined airways: impact of upper airway on lower airway. Curr Opin Otolaryngol \& 
Head and Neck Surg 2010, 18:1520.

4. Bousquet J, Vignola AM, Demoly P. Links between rhinitis and asthma. Allergy 2003: 58: 691-706.

5. Cruz A. The "united airways" require an holistic approach to management. Allergy 2005; 60: 871-874.

6. Antonicelli L, Braschi MC, Bresciani $M$, et al. The complex link between severity of asthma and rhinitis in mite allergic patients. Respir Med 2013; 107: 23-29.

7. Agresta MF, Saranz RJ, Lozano A, Lozano NA. Relación entre rinitis y asma: ¿Está todo dicho?. Revista de la Facultad de Ciencias Médicas 2014; 71(2):111-121.

8. Giavina-Bianchi $P$, Vivolo Aun $M$, Takejima P, Kalil J, Câmara Agondi R. United airway disease: current perspectives. Journal of Asthma and Allergy 2016:9; 93-100.

9. Saranz RJ. Vías aéreas unidas e impacto recíroco entre rinosinusitis y asma. Alergia Immunol Clin 2002;19:71-77

10. Saranz RJ, Lozano A, Alvarez JS, Croce VH. Factors associated to bronchial hyperresponsiveness in children and adolescents with allergic rhinitis. J Allergy Clin Immunol 1997; 99 (1): S 418.

11. Kim SW, Han DH, Lee SJ, et. al. Bronchial hyperresponsiveness in pediatric rhinitis patients: The difference between allergic and nonallergic rinitis. Am J Rhinol
12. Ianiero L, Saranz RJ, Lozano NA, Lozano A, Sasia LV, Ramírez M, Cuestas E. Análisis de la curva flujo-volumen en niños y adolescentes con rinitis alérgica sin asma. Arch Argent Pediatr 2013; 111(4): 322-327.

13. Ciprandi G, Cirillo I. The lower airway pathology of rhinitis. $J$ Allergy Clin Immunol 2006; 118: 1105-1109.

14. Chawes BLK. Upper and lower airway pathology in young children with allergic and non-allergic rhinitis. Dan Med Bull 2011; 58(5): 1-23.

15. Wang W, Xian M, Xie Y, Zheng J, Li J. Aggravation of airway inflammation and hyperresponsiveness following nasal challenge with Dermatophagoides pteronyssinus in perennial allergic rhinitis without symptoms of asthma. Allergy 2016; 71: 378-386.

16. Bertelsen RJ, Lødrup Carlsen KC, Carlsen $\mathrm{K}-\mathrm{H}$. Rhinitis in children: Co-morbidities and phenotypes. Pediatr Allergy Immunol 2010: 21: 612-622.

17. Togias A. Mechanisms of noselung interaction. Allergy 1999;54(Suppl 57):94-105.

18. Pasalacqua G, Ciprandi G, Canonica GW. The nose-lung interaction in allergic rhinitis and asthma: united airways disease. Curr Opin Allergy Clin Immunol 2001;1:7-13.

Allergy 2013; 27: 63-68. 
19. Simon RA. The allergy-asthma connection. Allergy Asthma Proc 2002;23:219-222.

20. Kariyawasam HH, Rotiroti G.

Allergic rhinitis, chronic rhinosinusitis and asthma: unravelling a complex relationship. Curr Opin Otolaryngol Head Neck Surg 2013, 21:79-86.

21. Denburg J. The nose, the lung and the bone marrow in allergic inflammation. Allergy 1999; 54:7380.

22. Braunstahl GJ. The unified immune system: Respiratory tractnasobronchial interaction mechanisms in allergic airway diseaseJ Allergy Clin Immunol 2005;115:142-8.

23. Rondon C, Campo P, Galindo L, Blanca-Lopez N, Cassinello MS, Rodriguez-Bada JL et al. Prevalence and clinical relevance of local allergic rhinitis. Allergy 2012;67: 1282-1288.

24. Campo P, Rondón C, Gould HJ, Barrionuevo E, Gevaert P, Blanca M. Local IgE in non-allergic rhinitis Clin Exp Allergy 2015;45: 872-881.

25. Bjermer $L$. The nose as an air conditioner for the lower airways. Allergy 1999;54, (suppl 57):26-30.

26. Zhang N, Van Crombruggen $\mathrm{K}$, Gevaert E, Bachert C. Barrier function of the nasal mucosa in health and type-2 biased airway diseases. Allergy 2016; 71: 295307.

27. Corren J, Adinoff AD, Irvin CG. Changes in bronchial responsiveness following nasal provocation with allergen. J Allergy Clin Immunol 1992;89:611-8.

28. Turkalj M, Živkovic J, Lipej M, Bulat Lokas S, Erceg D, Ante Anzic SA et al. The effect of mouth breathing on exercise induced fall in lung function in children with allergic asthma and rhinitis. International Journal of Pediatric Otorhinolaryngology 2016; 86: 5356.

29. Sluder G. Asthma as a nasal reflex. JAMA 1919; 73:589-591.

30. Gottlieb MS. Relation of intranasal sinus disease in the production of asthma. JAMA 1925; 85:105-109.

31. Undem BJ, McAlexander M, Hunter DD. Neurobiology of the upper and lower airways. Allergy 1999;54:8193.

32. Irvin CG. Sinusitis and asthma: an animal model. J Allergy Clin Immunol 1992;90: 521-5.

33. McFadden Jr ER. Nasal-sinuspulmonary reflexes and bronchial asthma. J Allergy Clin Immunol 1986;78:1-5.

34. Kaufman J, Wright G. The effect of nasal and nasopharyngeal irritation on airway resistance in man. Am Rev Respir Dis 1969;100:626-630.

35. Yan K, Salome C. The response of the airways to nasal stimulation in asthmatics with rhinitis. Eur $\mathrm{J}$ Respir Dis 1983;128(suppl):105109

36. Schumacher MJ, Cota KA, Taussig LM. Pulmonary response to nasal challenge testing of atopic subjects 
with stable asthma. J Allergy Clin Immunol 1986; 78:30-35.

37. Littell NT, Carlisle CC, Millman RP, et al. Changes in airway resistance following nasal provocation. Am Rev Respir Dis1990; 141:580-583

38. de Benedictis, FM, Bush A. Rhinosinusitis and asthma: Epiphenomenon or causal association?. Chest 1999; 115:550-556.

39. Fontanari P, Zattara-Hartmann MC, Burnet $\mathrm{H}$, Jammes $\mathrm{Y}$. Nasal eupnoeic inhalation of cold, dry air increases airway resistance in asthmatic patients. Eur Respir J 1997;10:2250-4.

40. Togias AG, Naclerio RM, Proud D, et al. Studies on the allergic and nonallergic nasal inflammation. J Allergy Clin Immunol 81:782-790, 1988.

41. Sanico A, Philip G, Proud D, Naclerio R, Togias A. Comparison of nasal mucosal responsiveness to neuronal stimulation in nonallergic and allergic rhinitis: effects of capsaicin nasal challenge. Clin Exp Allergy 1998;28:92-100.

42. Cingi C, Muluk NB, Cobanoglu B, Çatli T, Dikici O. Nasobronchial interaction. World J Clin Cases. 2015;3(6):499-503.

43. Baraniuk JN, Merck SJ. Nasal reflexes: implications for exercise, breathing, and sex. Curr Allergy Asthma Rep. 2008;8(2):147-153.

44. Bardin $P$, VanHeerden B, Joubert $\mathrm{J}$. Absence of pulmonary aspiration of sinus contents in patients with asthma and sinusitis. J Allergy Clin Immunol 1990;86:82-88.

45. Brugman $S$, Larsen $G$, Henson $P$, Honor J, Irvin C. Increases lower airways responsiveness associated with sinusitis in a rabbit model. Am Rev Respir Dis 1993;147:314-320.

46. Eifan AO, Durham SR. Pathogenesis of rhinitis. Clin Exp Allergy 2016; 46:1139-1151.

47. Ciprandi G, Caimmi D, Miraglia Del Giudice M, La Rosa M, Salpietro C, Marseglia GL. Recent developments in united airways disease. Allergy Asthma Immunol Res. 2012;4(4):171-177.

48. Foresi A, Leone C, Pelucchi A, Mastropasqua B, Chetta A, D'Ippolito R, Marazzini L et al. Eosinophils, mast cells and basophils in induced sputum from patients with seasonal allergic rhinitis and perennial asthma: Relationship to methacoline responsiveness. J Allergy Clin Immunol 1997;100:58-64.

49. Braunsthal GJ, Overbeek S, KleinJan A, Prins JB, Hoogsteden $\mathrm{H}$, Fokkens W. Nasal allergen provocation induces adhesion molecules expression and tissue eosinophilia in upper and lower airways. J Allergy Clin Immunol 2001;107:469-476.

50. Braunstahl GJ, Overbeek SE, Fokkens WJ, Kleinjan A, McEuen et al. Segmental bronchoprovocation in allergic rhinitis patients affects mast cell and basophil numbers in nasal and 
bronchial mucosa. Am J Respir Crit

Care Med 2001;164:858-865.

51. Saito H, Howie K, Wattie J,

Denburg A, Ellis R, Inman MD, et

al.Allergen-induced murine upper

airway inflammation: local and

systemic changes in murine

experimental allergic rhinitis.

Immunology 2001;104:226-34.

52. Xie JX, Xi Y, Zhang Q, et al. An

intratracheal challenge murine

model of asthma: can bronchial

inflammation affect the nose?

Allergy Asthma Immunol Res

2015;7:76-82.

53. Bresciani M, Paradis L, Des

Roches A, Vernhet H, Vachier I,

Godart Ph, Bousquet J, Chanez P.
Rhinosinusitis in severe asthma. J Allergy Clin Immunol 2001;107:7380.

54. Smurthwaite L, Durham SR. Local $\lg$ E synthesis in allergic rhinitis and asthma. Curr Allergy Asthma Rep 2002; 2:231-8.

55. van Zele $T$, Gevaert $P$, Holtappels $G$, van Cauwenberge P, Bachert C. Local immunoglobulin production in nasal polyposis is modulated by superantigens. Clin Exp Allergy 2007;37:1840-7.

56. Rondón C, Bogas G, Barrionuevo E, Blanca M, Torres MJ, Campo P. Nonallergic rhinitis and lower airway disease. Allergy 2017; 72 : 24-34. 